\title{
RETRACTION
}

\section{Retraction notice to: M1 macrophage-derived exosomes moderate the differentiation of bone marrow mesenchymal stem cells}

TAILIN WU1,\#; XIANG ZHOU2,\#; CANHUA YE ${ }^{1}$; WenCAN LU ${ }^{1}$; HAITAO LIN ${ }^{1}$; YANZHE WEI ${ }^{1}$; ZeKAI KE ${ }^{1}$; ZhenGJI HUANG ${ }^{1}$; JIANZHOU LUO ${ }^{1}$; HUIREN TAO ${ }^{1}$; CHUNGUANG DUAN $1{ }^{1}{ }^{*}$

${ }^{1}$ Shenzhen University General Hospital, Shenzhen, 518055, China

${ }^{2}$ Luoyang Central Hospital Affiliated to Zhengzhou University, Luoyang, 417000, China

The published article titled "M1 macrophage-derived exosomes moderate the differentiation of bone marrow mesenchymal stem cells", has been retracted from journal BIOCELL, Vol.46, No.2, 2022, pp.495-503.

\section{DOI: $10.32604 /$ biocell.2022.015214}

URL: https://www.techscience.com/biocell/v46n2/45109

The authors declare that due to the following reasons, they have to retract the published article from BIOCELL:

1. The authors retract this article owing to recently uncovered discrepancies in Figure 4 that were obtained. They have discovered that expression level of BMP2 between M0 and M1 has no significant difference according to their new verified results.

2. The authors cannot duplicate the results again on the same experimental conditions. All these issues decrease confidence in the integrity of the experimental findings reported.

After a comprehensive verification, in order to ensure the authenticity of the data and minimize the negative impact on readers, Tech Science Press has decided to accept the request for Retraction. The authors apologize for the Retraction and assume full responsibility for the consequences of what the Retraction has caused.

Tech Science Press is fully committed to enforcing strict ethical policies and following the COPE Retraction Guidelines. The essential condition of submitting a manuscript for publication in BIOCELL is that authors must explicitly declare that their works are original and have not been previously published elsewhere. Reuse of any data should be appropriately cited. To set the record straight, we hereby announce the Retraction of the paper, and would like to sincerely apologize to our readers for any inconvenience this may have caused. 